\title{
Vom Problem mit der Bestimmung des Fallproblems
}

\begin{abstract}
Herausforderungen bei der interaktiven Fallkonstitution als ein Kernprozess professioneller Praxis im Kontext (möglicher) Kindeswohlgefährdungen
\end{abstract}

Sozialarbeitende haben beim Auftreten von Erziehungs- und Entwicklungsproblemen in Familien oder auch bei potenziellen Kindeswohlgefährdungen mit konkreten realen Fällen zu tun. Ausgangspunkt des Beitrages ist die These, dass der Fall nicht objektiv gegebenen ist, sondern in der organisational gerahmten Interaktion zwischen den Klienten und Klientinnen und den Professionellen Sozialer Arbeit hergestellt wird.

$\mathrm{D}$ iese interaktive Fallkonstitution wird hier als ein Kernprozess professioneller Praxis - und damit auch der professionellen Urteilsbildung verstanden. Anhand der Befunde aus einer qualitativen Studie wird aufgezeigt, dass die Fallkonstitution eine voraussetzungsvolle und folgenreiche Herstellungsleistung ist und welche Herausforderungen damit einhergehen.

\section{Wer hat welche Probleme und was ist zu tun?}

Für Angehörigen von Professionen verweist der Fall auf ein aktiv zu lösendes Problem und er stellt den zentralen Modus ihres Denkens und Handelns dar. Fälle liegen aber nicht einfach als gegebene Bezugsgrössen des professionellen Handelns vor. Auf der Basis der Re-

\begin{tabular}{l} 
Cornelia Rüegger \\
$\begin{array}{l}\text { FHNW University of Applied Sciences and Arts Northwestern } \\
\text { Switzerland, } \\
\text { Olten, Schweiz } \\
\text { *1968; Dr. des., Sozialwissenschafterin und Sozialarbeiterin MA. } \\
\text { Wissenschaftliche Mitarbeiterin im Institut für Professionsfor- } \\
\text { schung und -entwicklung an der HSA FHNW. } \\
\text { cornelia.rueegger@fhnw.ch }\end{array}$ \\
\hline
\end{tabular}

Zusammenfassung Der Beitrag geht der Frage nach, wie Sozialarbeitende ein Wissen darüber entwickeln, was die Fallproblematik ausmacht und welcher Hilfe es bedarf. Es zeigt sich, dass die Fallkonstitution eine komplexe Herstellungsleistung ist und welche Herausforderungen und Fallstricke damit einhergehen. Dabei sticht die Selektivität bezüglich der verarbeiteten Informationen ebenso hervor wie die Bedeutung organisational vorgegebener Relevanzen.

Schlüsselwörter Fallkonstitution, Fallwissen, Soziale Diagnostik, Arbeitsbeziehung, Kindeswohlgefährdung levanzordnungen der jeweiligen Profession sind sie das Produkt der Konstitutionsleistungen von Professionellen, die aus einem Sachverhalt erst einen Fall herstellen (Bergmann 2014). In der Sozialen Arbeit wird die entsprechende Phase als soziale Diagnostik oder Fallverstehen bezeichnet. Dabei wird kontrovers diskutiert (bspw. Kunstreich et al. 2003), wie Professionelle und ihre Klienten und Klientinnen zu einer Einschätzung darüber kommen sollen, was der Fall ist, oder wie es BurkhardMüller (1997) formulierte: wer hat welche Probleme und was ist aus fachlicher Sicht zu tun?

Vor diesem Hintergrund werden in der Praxis Sozialer Arbeit tagtäglich auf der Basis von Schriftstücken, Hausbesuchen und im Gespräch mit den Klienten und Klientinnen in mehr oder weniger bewussten sozialdiagnostischen Prozessen (Rüegger et al. 2019) Falldeutungen mit entscheidungsrelevanten Kategorien produziert. Dies erfordert Herstellungsleistungen unterschiedlicher Art (auch Hall et al. 2003; Messmer und Hitzler 2007; Wolff 1983) die mitbestimmt sind durch:

- die Sozialarbeitenden und ihre Praktiken im Umgang mit diagnostischen Instrumenten (Ackermann 2021); impliziten Logiken (Schmidt 2012), Deutungsmuster (Hüttemann 2008); Erfahrungswissen, Intuition (Bastian et al. 2018; Becker-Lenz et al. 2017); alltägliche und professionelle Wissensbestände (Klomann et al. 2019; Rüegger et al. 2019); Moralvorstellungen (White 2003); professionelle Einstellungen und deren Einfluss auf die Beurteilung der Kindeswohlgefährdung (Gautschi 2021) u. a. m.,

- die Klienten und Klientinnen und ihre Erfahrungen im Umgang mit Hilfesystemen (Maeder und Nadai 2004) 


\section{Extrablick: Urteilsbildung in der Sozialen Arbeit}

sowie ihre Muster und Strategien der Problempräsentation (Rüegger 2021a) u. a. m.,

- die organisationalen Rahmenbedingungen, wie z. B. organisationale Konventionen und Routinen, Ressourcen für die Fallarbeit, Formulare, Klienten- und Klientinnen-Klassifizierungen (Bauer 2010; Busse et al. 2016; Rüegger et al. 2019); Formen interprofessioneller und interinstitutioneller Zusammenarbeit (Silkenbeumer und Thieme 2019); Angebots- und Versorgungsstruktur im jeweiligen Hilfesystem (Hansjürgens 2018; Rüegger et al. 2017).

Die Bestimmung der Fallproblematik in der Praxis stützt sich folglich nicht allein auf die Anwendung wissenschaftlichen Wissens auf die praktische Problemstellung hin. Die Praxis Sozialer Arbeit agiert vielmehr in einem Spannungsfeld von sozialstattlichem Auftrag bzw. organisational vorgeprägten Hilfeleistungen, den Anliegen ihrer Klienten und Klientinnen und professionellem Handlungsverständnis (auch Bommes und Scherr 2012). Den Sozialarbeitenden stellt sich hierbei die Herausforderung, die verschiedenen Wissensbestände im Hinblick auf die praktische Problemlösung und die Frage der angemessenen Hilfe aufeinander zu beziehen und zu relationieren (Dewe et al. 1992) und dies in Kooperation mit den Klienten und Klientinnen. Das ist kein trivialer Prozess, dessen Hergang bisher zudem wenig untersucht ist. In einer qualitativ-explorativen Studie ${ }^{1}$ (Rüegger 2021a) zu den Prozessen und Praktiken der Fallkonstitution wurde deshalb der Frage nachgegangen, wie das fallrelevante Wissen in der Interaktion zwischen Sozialarbeitenden und ihrer Klienten und Klientinnen „,in actu“ generiert, verschränkt und in Bezug auf Fragen der konkreten Hilfe handlungswirksam gemacht wird. Im Fokus stehen hierbei Fälle ${ }^{2}$, die in der Schulsozialarbeit, der der Sozialpädagogischen Familienbegleitung und einem Abklärungsdienst für Kindeswohlabklärungen angesiedelt sind und bei denen ein Verdacht auf eine Kindeswohlgefährdung im Raum steht.

\section{Wie wird der Fall zum Fall?}

Anhand der Fallanalysen wird sichtbar, dass der Prozess der Fallkonstitution mit einer Preconstruction beginnt, die der Interaktion mit den Klienten und Klientinnen vorausgeht. Im Fall aus der Schulsozialarbeit wird in der Preconstruction eine organisationsbezogene Problemwahrnehmung und entsprechend selektive Rahmung und Eingrenzung des Falles deutlich. Innerhalb der organisationsbezogenen Fallschablone zeigen sich oberflächliche Deutungsprozesse des Sozialarbeiters auf der Basis von beruflichem Erfahrungswissen und kulturalistischen Deutungsmustern. Die frühe Verortung und
Reduktion der komplexen Ausgangslage der beteiligten Familie in der Problemformulierung „Erziehungsstil Mutter" erweist sich auf seiner hidden agenda ${ }^{3}$ über den untersuchten Fallverlauf hinweg stabil und interventionswirksam in der Auswahl der Mittel und Methode (auch Becker-Lenz et al. 2015).

\section{Produktion des Falles in der Interaktion - zur Relevanz der Gesprächseröffnung}

Nach der Preconstruction kommt es mit Beginn des Erstgespräches zur Produktion des Falles in der Interaktion mit den Klienten und Klientinnen. In den untersuchten Gesprächen lassen sich verschiedene Handlungssegmente unterscheiden, in denen von den Sozialarbeitenden segmentspezifische Handlungsaufgaben kommunikativ bearbeitet werden. Für die Fallkonstitution ist vor allem die Gesprächseröffnung sowie die Problemschilderung und Exploration relevant.

Mit der Gesprächseröffnung bearbeiten die Sozialarbeitenden zunächst die Aufgabe, das Gespräch als organisational gerahmtes professionelles Gespräch mit einem bestimmten Zweck und Ziel zu konstituieren. Darüber wird auch die Beziehungsgestaltung aufgenommen, die organisationale Einbettung der Fallkonstitution angelegt, die Rolle als Klient_in und Sozialarbeiter_in eingerichtet sowie der Einstieg zur Problemexploration inhaltlich vorbereitet. Zum Ausdruck kommen kommunikative Praktiken der Sozialarbeitenden, die den Interaktionsteilnehmenden in verschiedener Hinsicht Orientierung geben, wie z. B. zum Handlungszweck des Gespräches oder zu sich als (Fach-) Person. Gleichzeitig wird deutlich, dass sich die Sozialarbeitenden über die Art und Weise der Thematisierung dieser orientierenden Aspekte darum bemühen, günstige Bedingungen für das nachfolgende Reden über Probleme der Lebensführung und die Inanspruchnahme Sozialer Arbeit zu schaffen. Zum Beispiel:

- die Inanspruchnahme der Sozialen Arbeit wird normalisiert

- Interventionen Sozialer Arbeit werden als Hilfe zur Erweiterung von Möglichkeiten und nicht als Eingriffshandeln dargestellt

- Sozialarbeitenden präsentieren sich als kompetente und erfahrene (Fach)Personen, die Expertise und Hilfe anbieten

- inhaltliche Engführung (Intransparenz) bezüglich jener Aspekte des konkreten (Abklärungs-)Auftrages, die aus Perspektive der Klienten und Klientinnen potenziell auch ein Risiko darstellen könnten

Zudem wird eine Reihe kommunikativer defensiver Praktiken sichtbar, wie zum Beispiel: 
- Vermeidung achtungsbedrohender Mitteilungsformen in der Gesprächseröffnung

- Vermeidung oder Abschwächung der eindeutigen Benennung heikler Themen oder Tatsachen (z. B. wie es zu diesem Gespräch gekommen ist; weshalb es geführt wird)

Diese defensiven Praktiken sind zu verstehen als ein Bemühen, die Klienten und Klientinnen als Interaktionsteilnehmende nicht in ein schlechtes Licht zu rücken und den Beziehungsaufbau dadurch zu erschweren oder gar zu verunmöglichen. Denn es geht bei den untersuchten Fallkonstitution immer auch um Sachverhalte und Situationen, die implizit mit Fragen von Schuld und Moral verbunden sind. Alle zuvor genannten Aufgaben der Gesprächseröffnung werden also auf eine Weise kommunikativ bearbeitet, die nicht nur das nachfolgende Reden über die Probleme begünstigt, sondern auch den Beziehungsaufbau möglichst wenig belastet.

\section{Produktion des Falles in der Interaktion - zur Problemschilderung und Exploration}

Die Analysen zeigen weiter, dass nach der Gesprächseröffnung die Problemschilderung und Exploration folgt. Sie beginnt mit der an die Klienten und Klientinnen gerichteten Aufforderung, allfällige Anliegen oder ihre Sicht auf einen bestimmten Sachverhalt zu schildern. Der thematische Zuschnitt ist durch die Sozialarbeitenden über die Gesprächseröffnung angelegt und es ist nicht gänzlich offen, worüber die Klienten und Klientinnen nun erzählen sollen. Die weitere Vorgehenswei$\mathrm{se}^{4}$ der Sozialarbeitenden innerhalb der Problemschilderung und Exploration ist jedoch sehr unterschiedlich: In der bereits erwähnten Fallkonstitution im Kontext der Schulsozialarbeit handelt es sich um eine strategisch ausgerichtete diagnostische Suchbewegung des Sozialarbeiters mit verstehenden Anteilen. Diese zielt aber weniger auf das Explorieren und Verstehen des Falles. Vielmehr geht es um das Auffinden von potenziellem Leidensdruck beim Klienten (Kindesvater).

Darüber versucht der Sozialarbeiter einen motivationalen Ansatzpunkt bei dem nicht an der Hilfe interessierten Klienten zu lokalisieren um seine hidden agenda zu bewerben und ein strategisches Bündnis einzurichten. Eine Art geteilte Problemsicht wird hergestellt in Bezug darauf, dass es einen ehelichen Paarkonflikt gibt. Doch der vorhandene Dissens zwischen dem Kindesvater und dem Sozialarbeiter bezüglich der Erklärung des Konfliktes in der Form von Falldeutungen macht der Sozialarbeiter im Gespräch nicht explizit, um die Entstehung des von ihm gewünschten Bündnisses nicht zu gefährden.
In einem starken Kontrast dazu steht die Arbeitsweise in der Problemschilderung und Exploration im Fall aus der Sozialpädagogischen Familienbegleitung. Sie lässt sich charakterisieren als ein Ausbreiten und Identifizieren von Fallthemen mit einer starken Orientierung des Sozialarbeiters an den Anliegen der Kindsmutter und ihren Verhaltensweisen und weniger bei Fragen des Kindeswohls. Mittels einer selektiven Thematisierung und De-Thematisierung einzelner Aspekte aus den Problemschilderungen der Klientin durch den Sozialarbeiter wird das Wissen zum Fall relationiert und der Fall als Fall für die Sozialpädagogische Familienhilfe hergestellt. Nochmals anders präsentiert sich die Problemschilderung und Exploration im Fall aus dem Abklärungsdienst. Die diagnostische Arbeitsweise des Sozialarbeiters lässt sich als ein Vorgehen charakterisieren, in dem einer Art diagnostischen „Spürens“ wie auch seinem Erfahrungswissen zentrale Bedeutung zukommt. Die Problemexploration dient vor allem der Erzeugung von Beschreibungswissen zum Fall auf der Basis der Problemschilderungen der Klientin, wobei der Sozialarbeiter ein spezielles Interesse an ihrem subjektiven Erleben und ihren biographischen Krisen zeigt. Auf dieser Basis bildet er sich ein im Gespräch nicht explizit kommuniziertes, günstiges Bild von der Klientin als „Mutter mit Herz“. Er produziert aber im interaktiven Geschehen keine expliziten Falldeutungen. Ein potenziell heikles, aber im Sinne der Abklärung des Kindeswohls wichtiges Thema (Verdacht von Gewalt des Partners gegenüber dem Kind) wird von ihm nicht angesprochen, um die Beziehungsebene nicht zu gefährden. Die Abklärung der Kindeswohlgefährdung bleibt durchgehend an der Oberfläche.

Trotz dieser Unterschiede lassen sich bestimmte Kernprozesse in der Herstellung von fallrelevantem Wissen herausarbeiten, nämlich das:

1. Hervorbringen von Fallwissen,

2. Selektionieren,

3. Verschränken/Relationieren sowie

4. Etablieren als geteiltes fallrelevantes Wissen (common ground).

Mittels dieser Kernprozesse wird zunächst das lebensweltliche Wissen der Klienten und Klientinnen zu ihren Anliegen und Problemen der Lebensführung hervorgebracht. Im Fall aus der Familienbegleitung und jenem aus dem Abklärungsdienst wird den Klienten und Klientinnen dabei ermöglicht, in sequenzieller Erstposition Wissen einzuführen und dabei eigene Relevanzsetzungen vorzunehmen. Die epistemische Autorität für dieses Wissen bleibt bei den Klienten und Klientinnen. Die Problemschilderung der Klienten und Klientinnen wird 


\section{Extrablick: Urteilsbildung in der Sozialen Arbeit}

durch die Sozialarbeitenden über Zuhören, Rückmeldesignale, Verstehensdokumentationen unterstützt und über Fragen aktiviert. Auch in den Problemschilderungen der Klienten und Klientinnen werden fallübergreifende Praktiken sichtbar (bspw. Problemaufwertungen, Verortung von Schuld bei anderen) und sie versuchen sich in ihren Problempräsentationen auf eine Weise darzustellen, dass sie ihr „Image“ (Goffman 1986) möglichst wahren können.

Aus den Problemschilderungen der Klienten und Klientinnen bzw. dem kommunikativen Fluss an so hervorgebrachtem Fallwissen, werden von den Sozialarbeitenden selektiv bestimmte Aspekte aufgegriffen und andere nicht thematisiert. Über dieses Selektionieren vollzieht sich ein Dimensionieren, eine Art Zuschneiden des Falles im Sinne eines Aufgreifens wie auch Weglassens bestimmter Aspekte aus den Erzählungen eigenerlebter Erfahrungen der Klient_innen durch die Sozialarbeitenden.

Diese Aspekte werden dann mehr oder weniger explizit mit den Wissensbeständen der Sozialarbeitenden verschränkt bzw. relationiert und darüber in alltagsweltliche Kategorien der Sozialarbeiter transferiert und transformiert wie auch in solche, die sich dem Fachwissen der Profession zuschreiben lassen. Im Fall aus der Sozialpädagogischen Familienbegleitung sind es Kategorien aus dem Fachwissen der Profession (z. B. als Fall von fehlendem „Grenzen setzen“), die aber den Fall zugleich in eine Passung mit dem Zuständigkeitsbereich der Organisation und ihren Unterstützungsangeboten bringen lassen. Es lässt sich stark vermuten, dass die beschriebene Selektion der Sozialarbeitenden und somit die Herstellung des Falles von diesem Ende her, also den Unterstützungsangeboten der Organisation, bedingt wird.

Über meist kooperativ verlaufende Verständigungsprozesse wird das so hergestellte Wissen zum Fall als geteiltes Wissen etabliert - oder noch Überzeugungsarbeit geleistet. Im ganzen Prozess bemühen sich die Sozialarbeitenden, wie schon in der Gesprächseröffnung, um kommunikative Formate, die achtungsbedrohende Mitteilungsformen möglichst vermeiden.

\section{Fazit und Diskussion}

Die skizzierten Befunde veranschaulichen, wie die Sozialarbeitenden in den untersuchten Fallkonstitutionen ein Wissen darüber entwickeln, was den Fall und seine Problematik ausmacht und welcher Hilfe es bedarf. Es wurde deutlich, dass die organisationale und interaktive Produktion des Falles eine voraussetzungsvolle, anspruchsvolle und folgenreiche Herstellungsleistung ist.
Auf Basis der empirischen Befunde werden aber auch Herausforderungen und Fallstricke professionellen Handelns in der Fallkonstitution sichtbar. Hierbei sticht die Selektivität der verarbeiteten Informationen ebenso hervor wie Blindstellen der Sozialarbeitenden bezüglich organisational vorgegebener Relevanzen. Durch die jeweilige Organisation wird die interaktive Fallkonstitution ermöglicht, aber eben auch eingeschränkt. Insofern sind die organisationsbezogenen Fallschablonen, wie sie sich in der Preconstruction noch vor einem Erstgespräch mit den Klienten und Klientinnen in den Akten der Sozialarbeitenden zeigen, ebenso wenig überraschend wie die Tatsache, dass der Fall in der Interaktion mit den Klienten und Klientinnen so hergestellt wird, dass er mit den Mitteln der Organisation bearbeitbar ist. Der Charakter des dabei erzeugten Fallwissens, das dann im Gespräch aktualisiert wird, ist den beteiligten Sozialarbeitenden selbst nur begrenzt bewusst. In Bezug darauf wird deutlich, dass die Praxis der Sozialen Arbeit vielfach nur begrenzt dem Anspruch einer wissenschaftlichen Fundierung - im Sinne einer bewussten und reflektierten Wissensverwendung - gerecht wird.

Die interaktiven Prozesse der Fallkonstitution sind aber nicht nur fachlich-inhaltlich herausfordernd. Deutlich wird die dynamische Verzahnung von diagnostischem Handeln und der Gestaltung der Arbeitsbeziehung in der Interaktion. Strukturell ist angelegt, dass sich Soziale Arbeit als gesellschaftlich institutionalisiertes Hilfesystem mit problematischen Formen der Lebensführung beschäftigt. Es geht hier also um eine Ist-Soll-Diskrepanz, die im Moment der Fallkonstitution in den Blick kommt und je nach Arbeitsfeld auch explizit ausgewiesen werden muss, um als unterstützungswürdig anerkannt zu werden oder allenfalls auch eine Kindeswohlgefährdung sichtbar zu machen. Empirisch zeigt sich, dass dies mit Prozessen der Herstellung von Hilfsbedürftigkeit der Erziehungsberechtigten oder auch der Schutzbedürftigkeit der Kinder und Jugendlichen sowie der meist impliziten Verhandlung von Schuld und Moral einher geht, was diese Prozesse der Fallkonstitution als situatives Beziehungsgeschehen so heikel macht (ausführlicher Rüegger 2021a). Die Sozialarbeitenden reagieren darauf mit den erwähnten defensiven Praktiken zur Vermeidung und Abschwächung (moralisierender) Inhalte. Oder wie der Sozialarbeiter aus dem Abklärungsdienst, der bestimmte Hinweise auf eine Kindeswohlgefährdung im Gespräch nicht direkt anspricht und so seine Diagnostik begrenzt, um eine (vermeintliche) Gefährdung der Arbeitsbeziehung zur Kindsmutter zu vermeiden, womit aber mögliche Leiden ihres Kindes nicht in den Blick kommen. 
Im Interview formuliert dieser Sozialarbeiter: „Verstehen braucht Beziehung und Vertrauen“. Dem ist mit Blick auf eine professionalisierte Praxis Sozialer Arbeit hinzuzufügen: (Fall-) Verstehen braucht Beziehung, Vertrauen, Wissen und Können der Professionellen und immer wieder der Reflexion der interaktiven Prozesse und Praktiken der Fallkonstitution und ihrer organisationalen Bedingungen.

Eingegangen. 20. April 2021

Angenommen. 14. Mai 2021

Funding. Open access funding provided by FHNW University of Applied Sciences and Arts Northwestern Switzerland.

Open Access. Dieser Artikel wird unter der Creative Commons Namensnennung 4.0 International Lizenz veröffentlicht, welche die Nutzung, Vervielfältigung, Bearbeitung, Verbreitung und Wiedergabe in jeglichem Medium und Format erlaubt, sofern Sie den/die ursprünglichen Autor(en) und die Quelle ordnungsgemäß nennen, einen Link zur Creative Commons Lizenz beifügen und angeben, $\mathrm{ob}$ Änderungen vorgenommen wurden.

Die in diesem Artikel enthaltenen Bilder und sonstiges Drittmaterial unterliegen ebenfalls der genannten Creative Commons Lizenz, sofern sich aus der Abbildungslegende nichts anderes ergibt. Sofern das betreffende Material nicht unter der genannten Creative Commons Lizenz steht und die betreffende Handlung nicht nach gesetzlichen Vorschriften erlaubt ist, ist für die oben aufgeführten Weiterverwendungen des Materials die Einwilligung des jeweiligen Rechteinhabers einzuholen.

Weitere Details zur Lizenz entnehmen Sie bitte der Lizenzinformation auf http://creativecommons.org/licenses/by/4.0/deed.de.

1. Das Datenmaterial wurden im Rahmen der Studie zu „Diagnostik und Arbeitsbeziehungen“ (z. B. Becker-Lenz et al. 2015) im Feld der Kinder- und Jugendhilfe erhoben. Als Datenbasis liegen aus sieben Fällen Tonaufzeichnungen von Gesprächen zwischen Sozialarbeitenden und ibrer Adressaten und Adressatinnen aus der Phase der Fallkonstitution sowie Protokolle teilnehmender Beobachtung vor. Ergänzt wurden diese Daten durch Dokumente, die im Rahmen der Fallkonstitution eingesetzt oder hergestellt wurden (Akten, Abklärungsbericht) und durch Daten, in denen sich ein organisationsspezifischer Blick auf den Fall manifestiert (Konzepte, Anamnesebögen), wie auch durch Interviews mit den Klient_innen und den Professionellen. Die Datenauswertung erfolgte mittels der Methode der objektiven Hermeneutik (Oevermann et al. 1979).

2. Im Laufe der Dissertationsstudie wurden Ergebnisse zu unterschiedlichen Aspekten bereits publiziert in Rüegger (2017, 2019, 2021b).

3. Für die Bezeichnung hidden agenda gibt es keine treffende deutsche Übersetzung. Sinngemäss geht es um eine Absicht, die aus taktischen Überlegungen verborgen bleibt.

4. Bezüglich der diagnostischen Arbeitsweisen gibt es eine Überschneidung mit dem gemeinsamen Forschungsprojekt mit Roland Becker-Lenz und Joel Gautschi (Becker-Lenz et al. 2015, 2017; Rüegger et al. 2019).

\section{Literatur}

Ackermann, T. (2021). Risikoeinschätzungsinstrumente und professionelles Handeln im Kinderschutz. Sozial Extra, 45, 42-48.

Bastian, P., Schrödter, M., Becker-Lenz, R., Gautschi, J., Grosse, M., Hunold, M., \& Rüegger, C. (2018). Bauchgefühle in der Sozialen Arbeit. In Sozialpädagogik, Kommission (Hrsg.), Wa(h)re Gefühle? Sozialpädagogische Emotionsarbeit im wohlfahrtsstaatlichen Kontext (S. 128-140). Basel: Beltz Juventa.

Bauer, P. (2010). Organisatorische Bedingungen der Fallkonstitution in der Sozialen Arbeit. Ein Literaturbericht. Zeitschrift für Pädagogik, 56(2), 249-266.

Becker-Lenz, R., Gautschi, J., \& Rüegger, C. (2015). Nicht-standardisiertes Wissen und nicht-methodisiertes Können in der sozialen Diagnostik. Einblick in eine empirische Analyse im Feld der Kinder- und Jugendhilfe. neue praxis. Zeitschrift für Sozialarbeit, Sozialpädagogik und Sozialpolitik, $45(3), 270-279$.

Becker-Lenz, R., Gautschi, J., \& Rüegger, C. (2017). Die Bedeutung von nicht-standardisiertem Wissen in der Diagnostik Sozialer Arbeit - Eine Fallanalyse zu „Erfahrungswissen“ und „Spüren" in einem Fall aus dem Kindesschutz. In H. Messmer (Hrsg.), Fallwissen. Wissensgebrauch in Praxiskontexten der Sozialen Arbeit (S. 115-154). Opladen: Budrich.

Bergmann, J. (2014). Der Fall als epistemisches Projekt. In J. Bergmann, G. Dausendschön-Gay \& F. Oberzaucher (Hrsg.), „Der Fall“c. Studien zur epistemischen Praxis professionellen Handelns (S. 423-440). Bielefeld: transcript.

Bommes, M., \& Scherr, A. (2012). Soziologie der Sozialen Arbeit. Eine Einführung in Formen und Funktionen organisierter Hilfe. Weinheim: Beltz Juventa.

Busse, St., Ehlert, G., Becker-Lenz, R., \& Müller-Hermann, S. (2016). Professionalität und Organisation. Wiesbaden: Springer VS.

Dewe, B., Ferchhoff, W., \& Radtke, F.-O. (1992). Das „Professionswissen“ von Pädagogen. Ein wissenstheoretischer Rekonstruktionsversuch. In B. Dewe, W. Ferchhoff \& F.-O. Radtke (Hrsg.), Erziehen als Profession. Zur Logik professionellen Handelns in pädagogischen Feldern (S. 70-91). Opladen: Leske + Budrich.

Goffman, E. (1986). Interaktionsrituale. Über Verhalten in direkter Kommunikation. Frankfurt a.M.: Suhrkamp.

Gautschi, J (2021). Urteile und Entscheidungen unter Unsicherheit in Kindeswohlabklärungen. Einflussfaktoren auf Fallbeurteilungen in einer multifaktoriellen, experimentellen Vignettenstudie. Pädagogische Hochschule Freiburg. Dissertation.

Hall, C., Juhila, K., Parton, N., \& Pöso, T. (2003). Constructing Clienthood in social work and human services. Interaction, identities and practices. London: Jessica Kingsley.

Hansjürgens, R. (2018). In Kontakt kommen. Analyse der Entstehung einer Arbeitsbeziehung in Suchtberatungsstellen. Baden Baden: Tectum.

Hüttemann, M. (2008). Was ist der Fall? Eine rekonstruktive Untersuchung diagnostisch relevanter Deutungsmuster von Fachkräften Sozialer Arbeit. Schweizerische Zeitschrift für Soziale Arbeit., 3(5), 32-56.

Klomann, V., Schermaier-Stöckl, B., Breuer-Nyhsen, J., \& Grün, A. (2019). Professionelle Einschätzungsprozesse im Kinderschutz. Forschungsbericht. Aachen: Katholische Hochschule NRW.

Kunstreich, T., Müller, B., Heiner, M., \& Meinhold, M. (2003). Diagnose und/oder Dialog? Ein Briefwechsel. Widersprüche. Zeitschrift für sozialistische Politik im Bildungs-, Gesundheits- und Sozialbereich, 23(88), 1-31.

Maeder, Ch, \& Nadai, E. (2004). Organisierte Armut. Sozialhilfe aus wissenssoziologischer Sicht. Konstanz: UVK.

Messmer, H., \& Hitzler, S. (2007). Die soziale Produktion von Klienten. Hilfeplangespräche in der Kinder- und Jugendhilfen. In B. Ludwig-Mayrhofer \& A. Sondermann (Hrsg.), Fallverstehen und Deutungsmacht. Akteure in der Sozialverwaltung und ibre Klienten (S. 41-74). Wiesbaden: Verlag Barbara Budrich.

Müller, B. (1997). Sozialpädagogisches Können. Ein Lehrbuch zur multiperspektivischen Fallarbeit. Freiburg im Breisgau: Lambertus.

Oevermann, U., Allert, T., Konau, E. \& Krambeck, J. (1979). Die Methodologie einer 'objektiven Hermeneutik' und ihre allgemeine forschungslogische Bedeutung für die Sozialwissenschaften. In: Soeffner, H-G (Hrsg.), Interpretative Verfahren in den Sozial- und Textwissenschaften (S. 352-434). Stuttgart: Metzler. 


\section{Extrablick: Urteilsbildung in der Sozialen Arbeit}

Rüegger, C. (2017). Die interaktive Herstellung des Falles und seiner Problematik in Gesprächen der Sozialen Arbeit. Erste Ergebnisse einer empirischen Studie zu Prozessen der Fallkonstitution im Feld der Kinder- und Jugendhilfe. In H. Messmer (Hrsg.), Fallwissen. Wissensgebrauch in Praxiskontexten der Sozialen Arbeit (S. 155-200). Opladen: Barbara Budrich.

Rüegger, C. (2019). Die interaktive Produktion des „Falles“ in der Sozialen Arbeit. Ein Blick auf kommunikative Prozesse und Praktiken im Ausgangspunkt (nicht)erwünschter Veränderungsprozesse. In E.-M. Graf, C. Scarvaglieri \& T. Spranz-Focasy (Hrsg.), Pragmatik der Veränderung. Problem- und lösungsorientierte Kommunikation in helfenden Berufen $(\mathrm{S}$. 239-263). Tübingen: Narr Franke Attempo.

Rüegger, C. (2021a). Fallkonstitution in Gesprächen Sozialer Arbeit. Prozesse und Praktiken der organisationalen und interaktiven Produktion des Falles. Wiesbaden: Springer.

Rüegger, C. (2021b). Zur Bestimmung des Falles und seiner Problematik im Ausgangspunkt professioneller Hilfe. Ein empirischer Einblick in die interaktive Herstellung des fallrelevanten Wissens im Prozess der Falldiagnostik. In D. Böhringer, S. Hitzler \& M. Richter (Hrsg.), Helfen. Situative und organisationale Ausprägungen einer unterbestimmten Praxis. Bielefeld: transript.

Rüegger, C., Ormanns, Y., Becker-Lenz, R., Gautschi, J., \& Rack, O. (2017). Gefährdungsmeldungen bezüglich älterer Menschen im Erwachsenenschutz: wer meldet was und warum (nicht)? Zeitschrift für Kindes- und Erwachsenenschutz, 6, 475-493.

Rüegger, C., Becker-Lenz, R., \& Gautschi, J. (2019). Zur Nutzung verschiedener Wissensformen in der Praxis Sozialer Arbeit. Erkenntnisse aus einer empirischen Analyse. In L. Hollenstein \& R. Kunz (Hrsg.), Kasuistik in der Sozialen Arbeit (S. 53-71). Opladen: Barbara Budrich.

Schmidt, F. (2012). Implizite Logiken des pädagogischen Blickes. Eine rekonstruktive Studie über Wahrnehmung im Kontext der Wohnungslosenhil $f e$. Wiesbaden: VS.

Silkenbeumer, M., \& Thieme, N. (2019). „Denn der Plan ist, dass Sarah mitgenommen wird “. Zur Fallkonstitution im interinstitutionellen Kooperationskontext ambulanter (sonderpädagogischer) schulischer Erziehungshilfe und Allgemeinem Sozialen Dienst. In Sozialpädagogik, Kommission (Hrsg.), Teilhabe durch"in*trotz Sozialpädagogik (S. 89-102). Weinheim, München: Beltz Juventa.

White, S. (2003). The social worker as a moral judge: blame, responsability and case formulation. In C. Hall, K. Juhila, N. Parton \& T. T. Pöso (Hrsg.), Constructing clienthood in social work and human services. Interaction, identities and practices (S. 177-192). London: Jessica Kingsley.

Wolff, St. (1983). Die Produktion von Fürsorglichkeit. Bielefeld: AJZDruck + Verlag. 